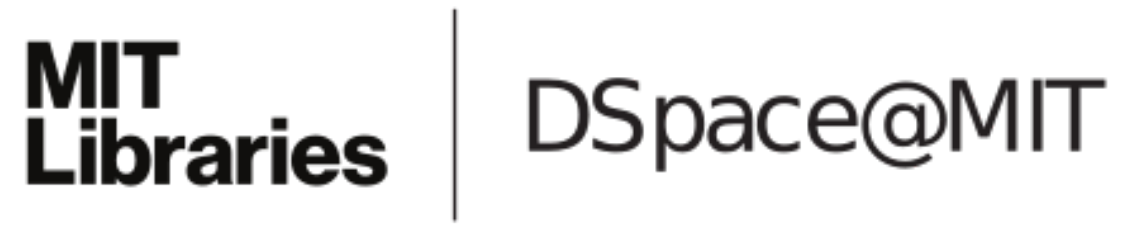

\author{
MIT Open Access Articles
}

Motion of a Solitonic Vortex in the BEC-BCS Crossover

The MIT Faculty has made this article openly available. Please share how this access benefits you. Your story matters.

Citation: Ku, Mark J.H., et al. "Motion of a Solitonic Vortex in the BEC-BCS Crossover." Phys. Rev. Lett. 113, 065301 (August 2014). (c 2014 American Physical Society

As Published: http://dx.doi.org/10.1103/PhysRevLett.113.065301

Publisher: American Physical Society

Persistent URL: http://hdl.handle.net/1721.1/88665

Version: Final published version: final published article, as it appeared in a journal, conference proceedings, or other formally published context

Terms of Use: Article is made available in accordance with the publisher's policy and may be subject to US copyright law. Please refer to the publisher's site for terms of use. 


\title{
Motion of a Solitonic Vortex in the BEC-BCS Crossover
}

\author{
Mark J. H. Ku, Wenjie Ji, Biswaroop Mukherjee, Elmer Guardado-Sanchez, \\ Lawrence W. Cheuk, Tarik Yefsah, and Martin W. Zwierlein \\ MIT-Harvard Center for Ultracold Atoms, Research Laboratory of Electronics, and Department of Physics, \\ Massachusetts Institute of Technology, Cambridge, Massachusetts 02139, USA
}

(Received 27 February 2014; published 4 August 2014)

\begin{abstract}
We observe a long-lived solitary wave in a superfluid Fermi gas of ${ }^{6} \mathrm{Li}$ atoms after phase imprinting. Tomographic imaging reveals the excitation to be a solitonic vortex, oriented transverse to the long axis of the cigar-shaped atom cloud. The precessional motion of the vortex is directly observed, and its period is measured as a function of the chemical potential in the BEC-BCS crossover. The long period and the correspondingly large ratio of the inertial to the bare mass of the vortex are in good agreement with estimates based on superfluid hydrodynamics that we derive here using the known equation of state in the BEC-BCS crossover.
\end{abstract}

DOI: 10.1103/PhysRevLett.113.065301

PACS numbers: 67.85.-d, 03.75.Kk, 03.75.Lm, 03.75.Ss

Solitary waves that do not spread as they propagate are ubiquitous in nonlinear systems, from classical fluids and fiber optics to superfluids and superconductors. These waves are localized objects with defined energy and mass, and as such they can be described as an effective single particle emerging from a many-body environment. This distinguishes them from larger-scale collective excitations such as shape oscillations of a superfluid, or from perturbative linear excitations such as phonons. Paradigmatic examples of solitary waves in superfluids are planar solitons that separate regions of differing phase, as well as vortex rings or single vortex lines [see Fig. 1(a)]. The direct creation of such localized and highly nonlinear objects "on demand" in ultracold quantum gases allows for an excellent dynamical probe of novel superfluids, such as strongly interacting Fermi gases [1] or spin-orbit coupled Bose-Einstein condensates [2,3].

In a recent experiment on fermionic superfluids at MIT [1], long-lived solitary waves were produced that featured a large ratio of inertial to bare (missing) mass of over 200, evidenced by an oscillation period over 15 times longer than the period for a single atom. The observed absorption images suggested the interpretation of the waves as planar solitons, but the longevity as well as the large effective mass ratio were unexpected for this type of defect [4-7]. Indeed, the nodal plane of a soliton is energetically more costly than the nodal line of a vortex, and planar solitons can decay into lower energy excitations via the snake instability, the undulation of the soliton plane [4]. Several recent works therefore suggested that these solitary waves are vortex rings [8-10]. For weakly interacting BoseEinstein condensates, solitons have been created [11,12] and observed to decay into vortex rings $[13,14]$. The latter further decay into a vortex-antivortex pair that eventually breaks up, leaving behind a single remnant vortex [15-17]. The exact process was recently elucidated in a discussion of apparent soliton oscillations observed in weakly interacting BECs $[18,19]$. In the case of strongly interacting fermionic superfluids, the understanding of such nontrivial dynamics presents a challenging nonequilibrium many-body problem $[8,20]$.

In this Letter, we investigate the nature of the long-lived solitary wave observed in Ref. [1] via tomographic imaging and identify the wave to be a solitonic vortex [15-17]. The term describes a vortex placed in an elongated geometry, such as the cigar-shaped superfluid of the present experiment, where the transverse Thomas-Fermi cloud radius $R_{\perp}$ is much smaller than the axial radius $R_{z}$. In this case, a vortex deforms the superfluid phase only in a restricted region of axial extent $\sim R_{\perp}$. The vortex together with the surrounding flow field thus constitutes an effective particle localized to within $R_{\perp} \ll R_{z}$. Its far-field phase profile resembles that of a dark soliton. In particular, the phase difference across a stationary solitonic vortex in the axial direction is $\pi$, the same as for a stationary dark soliton. A vortex moving in the axial direction at a critical speed converts into a gray, moving soliton [17]. In the tightly confining limit where $R_{\perp}$ approaches $\xi$, the vortex core size, a stationary solitonic vortex becomes energetically degenerate with a dark soliton $[16,17]$. In the limit of an isotropic system where $R_{\perp} \sim R_{z}$, one recovers a conventional vortex. A solitonic vortex thus represents the link between the topologically protected excitations in one and three dimensions. The precessional motion of the vortex, projected onto the long axis of the cigar-shaped atom cloud, appears as the oscillation of a particle of inertial mass $M^{*}$ and bare mass $M$. As we show below, the bare mass scales as the missing mass inside the vortex core, $M \propto m n \xi^{2} R_{\perp} \mathcal{L}$, while the inertial mass $M^{*} \propto m n R_{\perp}^{3} / \mathcal{L}$ is proportional to the volume $R_{\perp}^{3}$ in which flow is perturbed by the vortex. Here, $n$ is the gas density and $\mathcal{L}=\ln \left(R_{\perp} / \xi\right)$ is a logarithmic correction that is on the order of 3 to 5 in our 


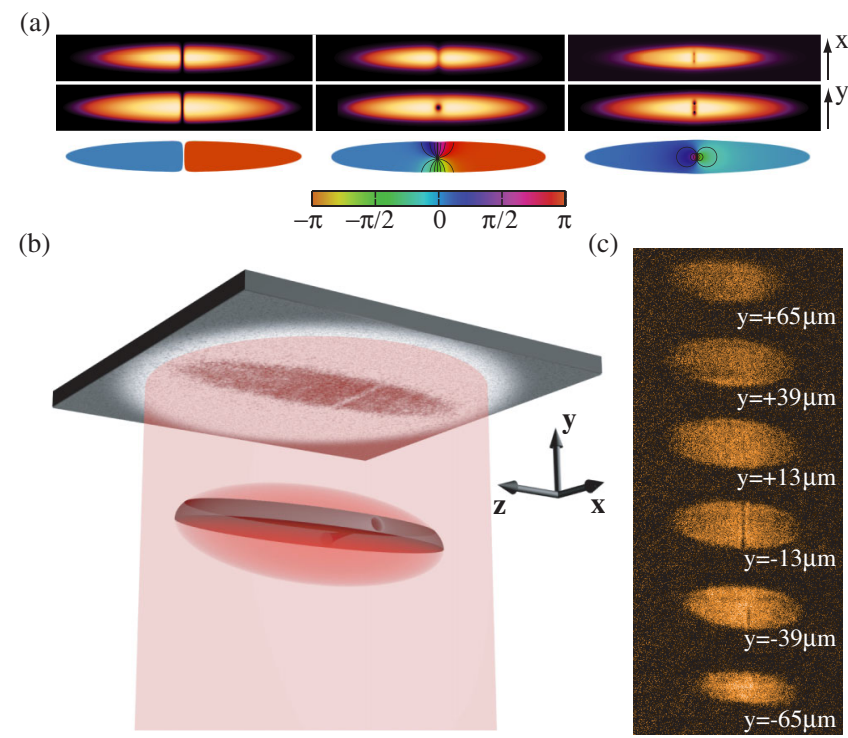

FIG. 1 (color online). (a) Examples of solitary waves in 3D Bose-Einstein condensates. Shown are simulated column density profiles in the $(z-x)$ plane (upper row), the local density of the cloud in a central layer in the $(z-y)$ plane (middle row), and the phase (lower row) for a soliton (left), a solitonic vortex (center), and a vortex ring (right). The images correspond to $\mu / \hbar \omega_{\perp}=7.31,7.14$, and 10.66. (b) Schematic of the experimental tomographic imaging technique. A partially masked optical pumping beam propagating along $z$ (not shown) selects a $23 \mu \mathrm{m}$ thick slice within the expanded atom cloud for absorption imaging along the vertical $y$ direction. (c) Tomography of a unitary fermionic superfluid of ${ }^{6} \mathrm{Li}$ atoms containing a solitary wave. Shown are density distributions of horizontal slices selected at different $y$ positions. Tomography reveals a single solitonic vortex.

experiment. Thus, $M^{*} / M \propto R_{\perp}^{2} / \xi^{2} / \mathcal{L}^{2}$, which can easily approach 200 for our experimental parameters, thus explaining the experimental findings in Ref. [1].

We create fermionic superfluids using a balanced mixture of the two lowest hyperfine states of ${ }^{6} \mathrm{Li},|1\rangle$ and $|2\rangle$. A Feshbach resonance allows us to tune the interparticle interactions from the limit of Bose-Einstein condensation of tightly bound molecules towards the regime of BCS superfluidity $[1,21]$. The atom cloud contains $1-10 \times 10^{5}$ atoms per spin state and is cigar shaped due to a tight radial confinement from an optical dipole trapping beam propagating along the (horizontal) $z$ direction, in combination with a weaker, harmonic confinement along $z$ provided by a magnetic field curvature. The radial and axial trapping frequencies are varied in the range of $\omega_{\perp} / 2 \pi \approx$ $55-75 \mathrm{~Hz}$ and $\omega_{z} / 2 \pi=5-23 \mathrm{~Hz}$. Gravity slightly weakens the trapping potential along the vertical $y$ direction, causing a residual anharmonicity and an anisotropy $\omega_{y} / \omega_{x}-1 \approx-5 \%$.

The solitary wave is created as in Refs. $[1,11,12,18]$ via phase imprinting, whereby one half of the superfluid is exposed to a blue-detuned laser beam for a duration that causes a phase shift of the order parameter close to $\pi$. To observe the magnitude of the superfluid wave function, we employ a rapid ramp to the BEC side of the Feshbach resonance during time of flight $[1,21,22]$. In addition to emptying out defects such as vortex cores [22], the ramp effectively increases the healing length $\xi$ of the superfluid to observable values (typically $\sim 20 \mu \mathrm{m}$ ). The observed width of the defect after the rapid ramp and time of flight thus does not reflect the in-trap width, which is expected to be on the order of one interparticle spacing $\sim 1 \mu \mathrm{m}$ [23]. Absorption images are taken along the vertical direction [see Fig. 1(b)].

In order to lift the ambiguity on the nature of the observed excitation, we employ a tomographic technique whereby only a chosen slice of the full atom cloud is imaged after time of flight [see Fig. 1(b)]. This method gives direct access to the local density of the 3D cloud. Tomography is achieved by optically pumping within $10 \mu \mathrm{s}$ all atoms outside the desired slice into hyperfine states that are off resonant with the imaging transition for state $|1\rangle$, predominantly state $|6\rangle$. The slice is selected by masking part of the optical pumping light with a thin wire, and projecting the wire's shadow onto the atom cloud. The slice thickness is measured to be 23(1) $\mu \mathrm{m}$ (=2 $\sigma$ of a Gaussian fit), comparable to the width of the observed solitary wave after time of flight, and about one sixth of the transverse cloud diameter after expansion. Since the imaging procedure is destructive, each run of the experiment provides a single slice at a given time of the defect's motion. Thanks to the high degree of stability of our experiment, reliable tomography can be built up from many repetitions of the experiment.

Representative tomographic images for the unitary fermionic superfluid are shown in Fig. 1(c), taken $1.6 \mathrm{~s}$ after the phase imprint. A line of depletion with about $40 \%$ contrast cuts across the entire cloud in one particular slice. This immediately demonstrates that the solitary wave is not a vortex ring. On average, only a specific one of the six slices imaged features the depletion. The strong depletion is thus not a planar soliton, as we interpreted in our previous paper [1] . Instead, our observation is consistent with a single, solitonic vortex. For the present experimental conditions we observe the vortex to be horizontal in every single repetition of the experiment. Due to the slight anisotropy of the trap, the vortex can minimize its energy by aligning along the short axis, while orientation along the longer, intermediate axis is unstable $[19,24,25]$. Slight tilts of the vortex into the vertical direction cause partial vortex lines to be detected in a given slice, as seen for slice position $y=-39 \mu \mathrm{m}$ in Fig. 1(c).

In a fully $3 \mathrm{D}$ setting where the radial cloud size $R_{\perp}$ is much larger than the vortex core size $\xi$, an off-center transverse vortex will undergo precessional motion along equipotential lines [26,27]. Tomographic imaging enables a measurement of the vortex position in the $z-y$ plane [see 
Figs. 1(b) and 1(c)]. The vortex's $z$ coordinate is readily obtained from the images. The $y$ coordinate is given by the position of the slice that contains the vortex (e.g., in Fig. 1(c), the vortex is observed at $y=-13 \mu \mathrm{m}$ ). In order to refine the determination of the $y$ position of the vortex, a histogram of occurrence as a function of the slice position is built up from several ( $\sim 7$ runs per slice position) repetitions of the experiment, and for each time of the vortex's motion. Representative images and density profiles of slices containing the vortex are shown in Fig. 2, along with histograms of the occurrence of vortex observations in each slice. The $z-y$ coordinates of the vortex lie on an ellipse with

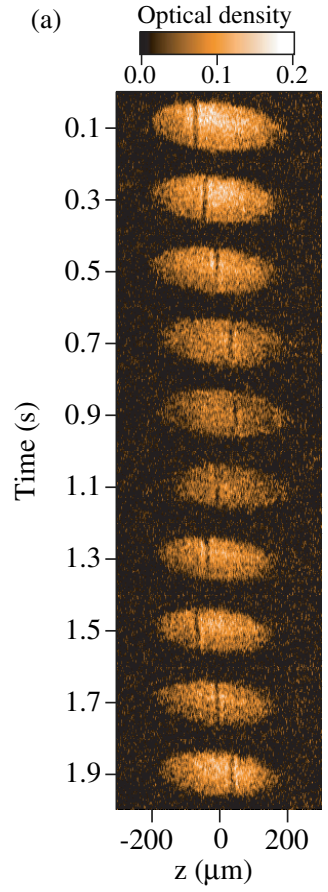

(d)

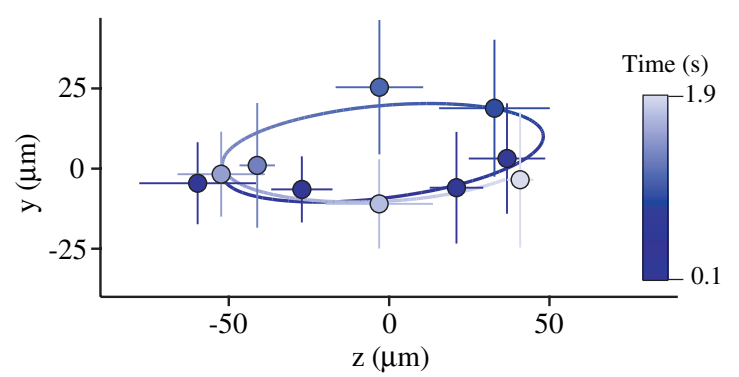

FIG. 2 (color online). Observation of vortex precession in a unitary fermionic superfluid via tomographic imaging. (a) Representative horizontal slices showing the oscillation of the vortex along the $z$ axis. Time $t=0 \mathrm{~s}$ marks $600 \mathrm{~ms}$ after the phase imprint. The $y$ position of each slice can be inferred from (c). (b) Density profiles normalized by the peak density, showing a depletion of typically $30 \%$ contrast. The solid line is a sine fit to the vortex positions (black dots). (c) Average occurrence of the vortex at a given $y$ position of the slice as function of time, showing the vortex oscillation along the $y$ axis. Red dots: average $y$ position of the vortex from Gaussian fit at the given time. Solid red line: sine fit. (d) Reconstructed precessional motion in the $z-y$ plane. the same aspect ratio (after expansion) as the atom cloud, as expected for vortex precession along equipotential lines.

The period of the vortex motion can be estimated from superfluid hydrodynamics and the equation of state in the BEC-BCS crossover [28]. Our Hamiltonian approach is analogous to that used to describe the motion of vortex rings in Ref. [29]. We take the vortex to be aligned in the transverse $x$ direction, and located at $\vec{r}_{0}=\left(y_{0}, z_{0}\right)$ in the $y-z$ plane. The free energy $E_{V}$ of the vortex is dominated by the kinetic energy of its flow field $\vec{v}=\hbar \nabla \phi / m_{B}$, where $\phi=\arctan \left(\left(y-y_{0}\right) /\left(z-z_{0}\right)\right)$ is the phase profile near the vortex, and $m_{B}=2 m$ the boson mass. One finds $E_{V} \approx$ $\int \frac{1}{2} m n v^{2} \mathrm{~d}^{3} r=\left(\pi \hbar^{2} m / m_{B}^{2}\right) n_{2 \mathrm{D}}\left(y_{0}, z_{0}\right) \ln \left(R_{\perp} / \xi\right)$ to logarithmic accuracy, i.e., in the limit $\ln \left(R_{\perp} / \xi\right) \gg 1$. Here, $n$ is the gas density, $n_{2 \mathrm{D}}$ is the column density along the vortex line, $R_{\perp}$ is the transverse Thomas-Fermi radius, much smaller than the axial radius $R_{z}$, and $\xi$ is the characteristic size of the vortex core. In the crossover we may take $\xi=\frac{1}{\sqrt{2}} \hbar / m_{B} c$, with $c$ the speed of sound, a definition that recovers the healing length in the BEC regime. At unitarity, this yields $\xi \approx 1 / k_{F}$ where $k_{F}=$ $\left(3 \pi^{2} n\right)^{1 / 3}$ is the Fermi wave vector, a reasonable estimate [23], especially within logarithmic accuracy.

The canonical momentum of the vortex along the axial $z$ direction is given by $P_{z}=\int m n v_{z} \mathrm{~d}^{3} r=\left(m / m_{B}\right)$ $\int \hbar n \partial_{z} \phi \mathrm{d}^{3} r$. Since $R_{\perp} \ll R_{z}$, the phase gradient is concentrated in the neighborhood of the vortex in a range of size $\sim R_{\perp}$ along the $z$ direction, allowing us to set $n(x, y, z) \approx n\left(x, y, z_{0}\right)$. The integral of $\partial_{z} \phi$ over the $z$ direction thus simply equals $\pi$ or $-\pi$, depending on whether the path runs along $y<y_{0}$ or $y>y_{0}$. One thus has $P_{z} \simeq$ $\left(m / m_{B}\right) \hbar \pi\left(\int_{-R_{\perp}}^{y_{0}} \mathrm{~d} y-\int_{y_{0}}^{R_{\perp}} \mathrm{d} y\right) n_{2 \mathrm{D}}\left(y, z_{0}\right)=\left(m / m_{B}\right) \hbar \pi$ $\int_{-y_{0}}^{y_{0}} \mathrm{~d} y n_{2 \mathrm{D}}\left(y, z_{0}\right)$. Assuming harmonic trapping and the local density approximation, we deduce the axial velocity of the vortex from Hamilton's equation

$$
\dot{z}_{0}=\frac{\partial E_{V}}{\partial P_{z}}=\frac{\partial E_{V} / \partial y_{0}}{\partial P_{z} / \partial y_{0}}=-\frac{\omega_{\perp}}{\omega_{z}} \Omega y_{0},
$$

and similarly $\dot{y}_{0}=\left(\omega_{z} / \omega_{\perp}\right) \Omega z_{0}$, with the angular frequency

$$
\frac{\Omega}{\omega_{z}}=\frac{2 \gamma+1}{8} \frac{\hbar \omega_{\perp}}{\mu} \ln \left(\frac{R_{\perp}}{\xi}\right) .
$$

Here, $\gamma \equiv(\mu / n)(\partial n / \partial \mu)$ is a polytropic index determined by the equation of state, and $\mu$ is evaluated at the vortex position. $\gamma=1$ in the BEC regime, while $\gamma=3 / 2$ at unitarity and in the BCS regime. The equations describe the precessional motion of the vortex with angular frequency $\Omega$ along an equipotential line of the trap with $\mu=$ const, i.e., $y_{0}^{2} / R_{\perp}^{2}+z_{0}^{2} / R_{z}^{2}=$ const. The result is identical to what one finds by equating the Magnus force [30] $h n_{2 \mathrm{D}} \hat{x} \times \dot{\vec{r}}_{0}$ to the force $-\nabla E_{V}$ acting on the vortex, and it generalizes the known result for vortex motion in 
trapped, weakly interacting Bose-Einstein condensates $[26,27]$ to superfluids with arbitrary equation of state. We find the inertial mass of the vortex [5]

$$
M^{*}=\frac{\partial P_{z}}{\partial \dot{z}_{0}}=\frac{\partial P_{z} / \partial y_{0}}{\partial \dot{z}_{0} / \partial y_{0}}=-\frac{4 \pi}{2 \gamma+1} \frac{n_{2 \mathrm{D}} R_{\perp}^{2}}{\ln \left(R_{\perp} / \xi\right)} m
$$

which is proportional to the total mass of atoms contained in the volume $R_{\perp}^{3}$, while the bare mass

$$
M=-\frac{\partial E_{V}}{\partial \mu} m=-\pi \frac{2 \gamma+1}{4 \gamma} n_{2 \mathrm{D}} \xi^{2} \ln \left(\frac{R_{\perp}}{\xi}\right) m
$$

is only proportional to the mass of "missing" atoms contained in the vortex core. Here we have used $\mu=\gamma m c^{2}=\gamma \hbar^{2} / 2 m \xi^{2}$. The ratio $M^{*} / M \propto R_{\perp}^{2} / \xi^{2} /$ $\left[\ln \left(R_{\perp} / \xi\right)\right]^{2}$ thus depends on the transverse size of the system and can become large. In contrast, the bare and inertial mass of a planar soliton are both on the order of the mass of "missing" atoms in the soliton plane, $\propto n \xi R_{\perp}^{2}$, and their ratio is bound to be on the order of unity in the crossover regime close to resonance. Using the experimental parameters of $\mu / \hbar \omega_{\perp} \approx 25-35$ [1], the hydrodynamical model yields a normalized vortex period $T_{V} / T_{z} \approx 11-15$ and effective mass ratio $M^{*} / M=130-220$, in close agreement with the measured values.

We have taken extensive data for the vortex period in the BEC-BCS crossover exploring a wide range of chemical potentials. Figure 3 shows the normalized period $T_{V} / T_{z}$ versus $\mu / \hbar \omega_{\perp}$ including data for several aspect ratios, interaction strengths, and atom numbers. Chemical potentials were extracted from the measured axial Thomas-Fermi radius of the cloud and the known axial trapping frequency. The inset in Fig. 3 shows the data from Ref. [1] along with the theoretical prediction for a fixed, characteristic atom number of $N / 2=3 \times 10^{5}$ per spin state, using the known equation of state in the BEC-BCS crossover [28]. The data are in good agreement with the approximate theory, from the BEC regime towards resonance and into the BCS regime. Corrections beyond logarithmic accuracy could be important as $\ln \left(R_{\perp} / \xi\right)$ is only $3-5$, but they are not known in the crossover beyond the weakly interacting BEC regime, and are the subject of debate [31]. Generally, there will be a contribution to the vortex's inertial mass from superfluid backflow, the Baym-Chandler mass [32]. For a strongly interacting Bose gas, quantum depletion localized inside vortex cores will modify the inertial and bare mass $[33,34]$. In the BCS regime, one expects a contribution due to fermions trapped in Andreev bound states inside the vortex core [35], the Kopnin mass [36]. For a molecular $\mathrm{BEC}$, the prediction from the Gross-Pitaevskii equation is $[26,27] \frac{\Omega}{\omega_{2}}=\frac{3}{8}\left(\hbar \omega_{\perp} / \mu\right)\left[\ln \left(R_{\perp} / \xi\right)+\frac{3}{4}\right]$ and is shown in Fig. 3 to agree well with the data.

An interesting future investigation concerns the early times a few milliseconds after the phase imprinting. Is the

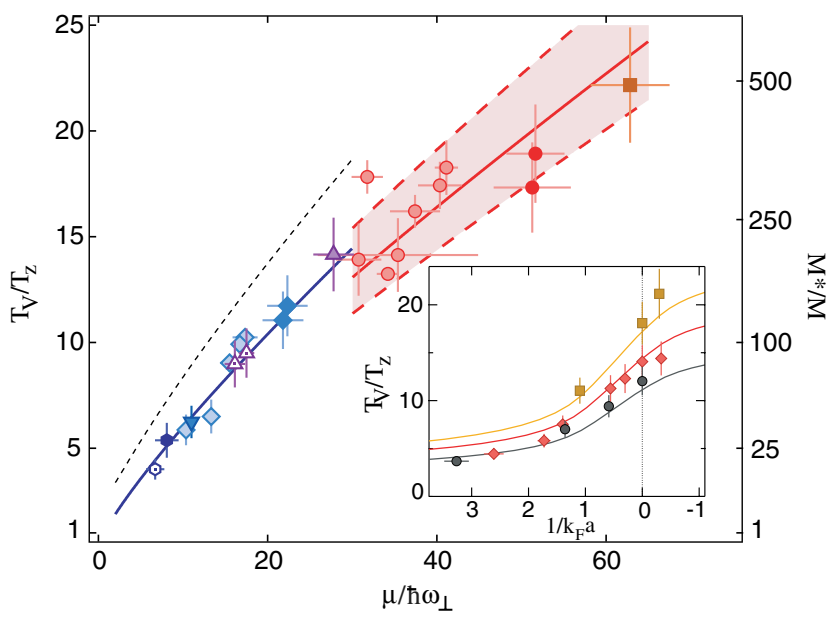

FIG. 3 (color online). Normalized period of the solitonic vortex $T_{V} / T_{z}$, as a function of the normalized chemical potential $\mu / \hbar \omega_{\perp}$. Experimental data are for magnetic fields $\mathrm{B}=850 \mathrm{G}$ (BCS side, square) and $832 \mathrm{G}$ (unitarity, circle), and for the BEC side at $800 \mathrm{G}$ (triangle), $760 \mathrm{G}$ (diamond), $740 \mathrm{G}$ (inverted triangle), and $700 \mathrm{G}$ (hexagon). $\omega_{z} / 2 \pi$ was $23 \mathrm{~Hz}$ (solid symbols), $10 \mathrm{~Hz}$ (framed), $5 \mathrm{~Hz}$ (dotted). Predictions in the BEC regime: solid blue line from Ref. [26], dashed black line from Ref. [27]. Red solid curve: hydrodynamic prediction on resonance, assuming $\xi=1 / k_{F}$. The error band is bounded by the choice $\xi=1 / 2 k_{F}$ (lower bound) and $\xi=2 / k_{F}$ (upper bound). Inset: $T_{V} / T_{z}$ as a function of the interaction parameter $1 / k_{F} a$, data from Ref. [1]. Square, diamond, and circle are for $\omega_{z} / 2 \pi=23,10$, and $5 \mathrm{~Hz}$, respectively. Atom numbers range from $N / 2=1 \times 10^{5}$ per spin state in the $\mathrm{BEC}$ regime to $3 \times 10^{5}$ around resonance. Solid curves: hydrodynamic prediction fixing $N / 2=3 \times 10^{5}$, and $\omega_{z} / 2 \pi=23 \mathrm{~Hz}$ (gold), $10 \mathrm{~Hz}$ (red), and $5 \mathrm{~Hz}$ (black).

single observed vortex a result of multiple decay processes, in which an initial planar soliton decays into a vortex ring, that further decays into vortex-antivortex pairs, followed by a "sling-shot" event [19] by which one of the vortices is ejected? Or does the phase imprint rather directly create vortices of a given circulation? For example, solitons that are slightly tilted with respect to the transverse direction can efficiently convert into solitonic vortices of one type of charge, removing the required angular momentum from a collective mode of the gas cloud [37].

In conclusion, we have implemented a tomographic imaging technique that allowed us to conclusively demonstrate that a long-lived solitary wave observed in our fermionic superfluid is a solitonic vortex. The vortex is topologically protected, explaining the long lifetime of the wave, and its theoretical inertial to bare mass ratio agrees with that found experimentally. Solitonic vortices can be expected to occur as persistent defects created via a KibbleZurek mechanism $[38,39]$, via phase imprinting $[18,19]$ or even via thermal excitations, as hinted at by the observation of thermally induced defects in Ref. [1]. They also correspond to the " $N$ "-shaped vortices created via rotation 
in Ref. [40], in the limit of zero rotation frequency (called " $S$ " shaped in Ref. [41]). Further studies on this topological excitation created "on demand" concern the interaction of multiple solitonic vortices in fermionic superfluids, a measurement of the current-phase relation of solitonic vortices [17], their contribution to flow resistance of the superfluid [42], and the observation of Andreev states bound to vortex cores [35].

We would like to thank David Huse, Lev Pitaevskii, and Joachim Brand for fruitful discussions. This work was supported by the NSF, the ARO MURI on Atomtronics, AFOSR PECASE, ONR, a grant from the Army Research Office with funding from the DARPA OLE program and the David and Lucile Packard Foundation.

Note added.-Recently, two related manuscripts [43,44] have been posted online.

[1] T. Yefsah, A. T. Sommer, M. J. H. Ku, L. W. Cheuk, W. Ji, W. S. Bakr, and M. W. Zwierlein, Nature (London) 499, 426 (2013).

[2] Y-J. Lin, K. Jiménez-García, and I. B. Spielman, Nature (London) 471, 83 (2011).

[3] A. L. Fetter, Phys. Rev. A 89, 023629 (2014).

[4] A. E. Muryshev, H. B. van Linden van den Heuvell, and G. V. Shlyapnikov, Phys. Rev. A 60, R2665 (1999).

[5] R. G. Scott, F. Dalfovo, L. P. Pitaevskii, and S. Stringari, Phys. Rev. Lett. 106, 185301 (2011).

[6] R. Liao and J. Brand, Phys. Rev. A 83, 041604 (2011).

[7] A. Cetoli, J. Brand, R. G. Scott, F. Dalfovo, and L. P. Pitaevskii, Phys. Rev. A 88, 043639 (2013).

[8] A. Bulgac, M. M. Forbes, M. M. Kelley, K. J. Roche, and G. Wlazłowski , Phys. Rev. Lett. 112, 025301 (2014).

[9] M. D. Reichl and E. J. Mueller, Phys. Rev. A 88, 053626 (2013).

[10] W. Wen, C. Zhao, and X. Ma, Phys. Rev. A 88, 063621 (2013).

[11] S. Burger, K. Bongs, S. Dettmer, W. Ertmer, K. Sengstock, A. Sanpera, G. V. Shlyapnikov, and M. Lewenstein, Phys. Rev. Lett. 83, 5198 (1999).

[12] J. Denschlag, J. E. Simsarian, D. L. Feder, C. W. Clark, L. A. Collins, J. Cubizolles, L. Deng, E. W. Hagley, K. Helmerson, W. P. Reinhardt, S. L. Rolston, B. I. Schneider, and W. D. Phillips, Science 287, 97 (2000).

[13] B. P. Anderson, P. C. Haljan, C. A. Regal, D. L. Feder, L. A. Collins, C. W. Clark, and E. A. Cornell, Phys. Rev. Lett. 86, 2926 (2001).

[14] Z. Dutton, M. Budde, C. Slowe, and L. V. Hau, Science 293, 663 (2001).

[15] J. Brand and W. P. Reinhardt, J. Phys. B 34, L113 (2001).

[16] J. Brand and W. P. Reinhardt, Phys. Rev. A 65, 043612 (2002).

[17] S. Komineas and N. Papanicolaou, Phys. Rev. A 68, 043617 (2003).
[18] C. Becker, S. Stellmer, P. Soltan-Panahi, S. Dörscher, M. Baumert, E. M. Richter, J. Kronjäger, K. Bongs, and K. Sengstock, Nat. Phys. 4, 496 (2008).

[19] C. Becker, K. Sengstock, P. Schmelcher, P. G. Kevrekidis, and R. Carretero-Gonzalez, New J. Phys. 15, 113028 (2013).

[20] P. Scherpelz, K. Padavić, A. Rançon, A. Glatz, I. S. Aranson, and K. Levin, arXiv:1401.8267.

[21] W. Ketterle and M. Zwierlein, Riv. Nuovo Cimento Soc. Ital. Fis. 31, 247 (2008).

[22] M. W. Zwierlein, J. R. Abo-Shaeer, A. Schirotzek, C. H. Schunck, and W. Ketterle, Nature (London) 435, 1047 (2005).

[23] A. Bulgac and Y. Yu, Phys. Rev. Lett. 91, 190404 (2003).

[24] A. A. Svidzinsky and A. L. Fetter, Phys. Rev. A 62, 063617 (2000).

[25] Following submission of the present work, this question was investigated numerically in Ref. [43], where the slight anharmonicity was shown to also play an important role in stabilizing the vortex orientation.

[26] E. Lundh and P. Ao, Phys. Rev. A 61, 063612 (2000).

[27] A. L. Fetter and J.-k. Kim, J. Low Temp. Phys. 125, 239 (2001).

[28] N. Navon, S. Nascimbene, F. Chevy, and C. Salomon, Science 328, 729 (2010).

[29] L. P. Pitaevskii, arXiv:1311.4693.

[30] P. Ao and D. J. Thouless, Phys. Rev. Lett. 70, 2158 (1993).

[31] E. B. Sonin, Phys. Rev. B 87, 134515 (2013).

[32] G. Baym and E. Chandler, J. Low Temp. Phys. 50, 57 (1983).

[33] A. L. Fetter, Phys. Rev. Lett. 27, 986 (1971).

[34] A. L. Fetter, Ann. Phys. (N.Y.) 70, 67 (1972).

[35] C. Caroli, P. G. De Gennes, and J. Matricon, Phys. Lett. 9, 307 (1964).

[36] N. B. Kopnin, JETP Lett. 27, 390 (1978);

[37] N. Parker, Numerical Studies of Vortices and Dark Solitons in Atomic Bose-Einstein Condensates, Ph.D. thesis, University of Durham, 2004.

[38] C. N. Weiler, T. W. Neely, D. R. Scherer, A. S. Bradley, M. J. Davis, and B. P. Anderson, Nature (London) 455, 948 (2008).

[39] G. Lamporesi, S. Donadello, S. Serafini, F. Dalfovo, and G. Ferrari, Nat. Phys. 9, 656 (2013).

[40] P. Rosenbusch, V. Bretin, and J. Dalibard, Phys. Rev. Lett. 89, 200403 (2002).

[41] S. Komineas, N. R. Cooper, and N. Papanicolaou, Phys. Rev. A 72, 053624 (2005).

[42] B. I. Halperin, G. Refael, and E. Demler, Int. J. Mod. Phys. B 24, 4039 (2010).

[43] G. Wlazłowski, A. Bulgac, M. M. Forbes, and K. J. Roche, arXiv:1404.1038.

[44] S. Donadello, S. Serafini, M. Tylutki, L. P. Pitaevskii, F. Dalfovo, G. Lamporesi, and G. Ferrari, Phys. Rev. Lett. 113, 065302 (2014). 